\title{
Estimating the influence of age-related changes in skin stiffness on tactile perception for static stimulations
}

\author{
Toru HAMASAKI*, Takahiro YAMAGUCHI* and Masami IWAMOTO* \\ *TOYOTA CENTRAL R\&D LABS., INC. \\ 41-1 Yokomichi, Nagakute, Aichi, 480-1192, Japan \\ E-mail: hamasaki@mosk.tytlabs.co.jp
}

Received: 2 November 2017; Revised: 7 December 2017; Accepted: 9 January 2018

\begin{abstract}
It is difficult to experimentally observe the influence of differences in individual skin properties of human fingers on tactile perceptions. During subjective experiments, many parameters, such as skin properties, the transmittance of nerve signals, and individual feelings are intricately mixed; therefore, it is difficult to identify which elements are affected and to what extent. It has not been determined how age-related changes in the stiffness of skin influence tactile perceptions. We developed a two-dimensional cross-section human fingertip finite element model based on previous studies. Then, we estimated the influence of age-related changes in skin stiffness on Merkel cells and tactile perceptions by using finite element analysis. Age-related changes in skin stiffness were described by changing skin material properties in the model. Simulations using a model involving the fingertip being contacted with a rigid flat plate or a 2-point indenter were performed. Using a rigid flat plate and standard skin material properties, the contact width between the finger pad and the object was $5 \mathrm{~mm}$. Meanwhile, the width changed from $5 \mathrm{~mm}$ to $4 \mathrm{~mm}$ when using a model of age-related changes of skin. Using a rigid 2-point indenter, the difference in the peak-to-valley of the Mises distribution around the Merkel cells indicated 2-point discrimination. Besides, the difference decreased by approximately $43 \%$ with changes in skin stiffness. These results indicated that age-related changes in skin stiffness influenced tactile perceptions.
\end{abstract}

Keywords : Tactile perception, Mechanoreceptor, Influence of skin stiffness, Aging, Finite element analysis

\section{Introduction}

Tactile perceptions provide us with physical information regarding the external world, especially the shape, material, and motion of objects. Moreover, this information allows us to control force and perform intended movements.

Human skin consists of three layers, the epidermis, dermis, and subcutaneous tissue (Johnson, 2002). At the interface between the epidermis and dermis, human skin has dermal papillae, which have a ridge-like shape. Each of the layers and dermal papillae has tactile receptors, which are mechanoreceptors (Vallbo and Johansson, 1984). A mechanoreceptor is a sensory receptor that responds to mechanical deformation of skin. The receptors are represented by Merkel cells, Meissner corpuscles, Ruffini endings, and Pacinian corpuscles. Merkel cells are located at the base of the dermal papillae and allow the perception of pressure and slow stimulus. Ruffini endings are within the dermis and allow the recognition of the stretching of skin. Meissner corpuscles are located at the top of dermal papillae and allow the perception of dynamical transverse deformation of the skin. Pacinian corpuscles are located within the subcutaneous tissue and help us recognize vibrations. Figure 1 shows a skin cross-section and the typical position where each receptor is placed.

Human fingers have a higher density of tactile receptors than others (Vallbo and Johansson, 1984). In addition, fingers have fingerprints on the finger pads. Therefore, fingers can obtain finer tactile information than other body parts. This finer perception enables us to perform detailed and accurate movements.

We are able to perceive tactile information through tactile receptors. However, the effects of the finger skin 


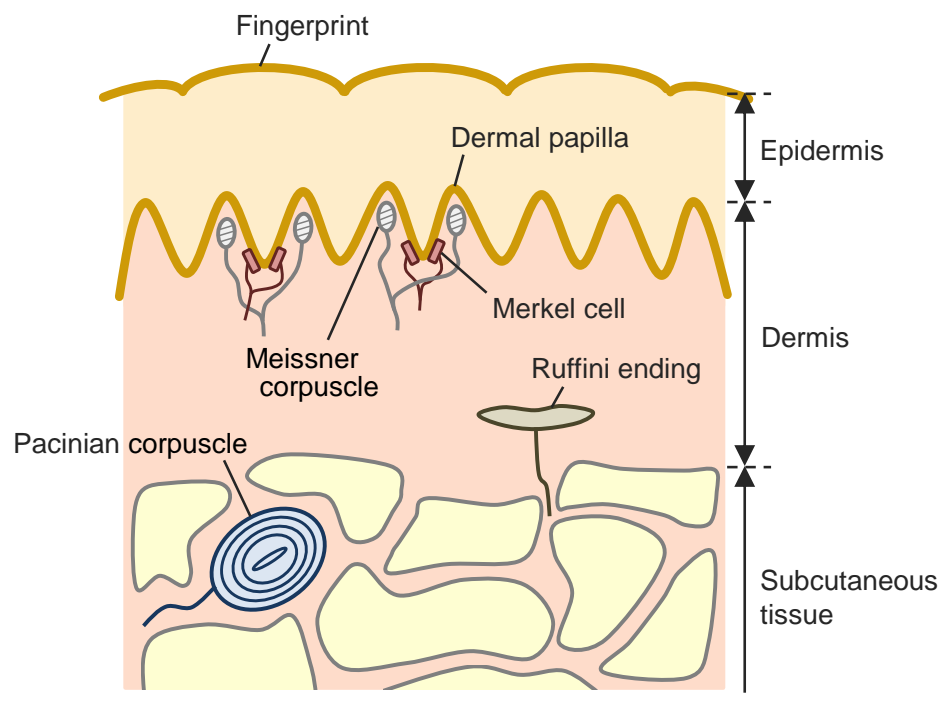

Fig. 1 Internal structure of the human skin and mechanoreceptors (i.e., Merkel cells, Meissner corpuscles, Ruffini endings, and Pacinian corpuscles). These receptors provide us with physical information of the external world, especially the shape, material, and motion of objects.

structure on tactile perception have not been clarified. It is difficult to experimentally observe changes in mechanical states such as stress and strain distributed around tactile receptors evoked by mechanical stimulus applied to the fingers. Therefore, finite element models of the finger have been used instead of in vivo experiments to estimate the stress and strain distribution around tactile receptors. Srinivasan and Dandekar (1996) developed a two-dimensional cross-sectional model of the finger perpendicular to a distal phalanx, and predicted the mechanical state around tactile receptors when the finger pad was in contact with an uneven corrugated rod. The study indicated that the strain energy density distribution and maximum principal strain had a trend similar to that of nerve discharge frequency. The results showed that the mechanical state and response of tactile receptors were estimated by finite element analysis. Using the calculation for simulating tactile motions of tracing an object with a step shape by using a three-dimensional finger shape model, the strain energy density distribution around tactile receptors also corresponded to nerve discharge (Dandekar et al., 2003).

Fingerprints and dermal papillae were not considered in the finger finite element models in the aforementioned studies. However, Maeno et al. (1998) and Gerling (2010) developed a two-dimensional finger cross-sectional model including fingerprints and dermal papillae. They compared the stress distributions around the tactile receptors using the model with or without fingerprints and dermal papillae. The results indicated that the distributions of the mechanical state including fingerprints and dermal papillae were higher than those of the simplified model. They suggested that the finger structure including the fingerprint and dermal papillae might enable tactile receptors to perceive the stimulus effectively.

Much data regarding tactile perceptions have been found by physiological and mechanical approaches such as those described here. Furthermore, changes in the skin with aging are well known (Saxon et al., 2010; Daly and Odland, 1979); for example, aging causes the epidermis to become stiff (Kuwazuru et al., 2008). Moreover, it is known that tactile perceptions change with aging and that elderly people have dull perceptions (Stevens, 1992; Carmeli et al., 2003). However, how age-related changes in skin stiffness affect tactile perceptions have not been elucidated with experiments (Wickremaratchi and Llewelyn, 2006; Bowden and McNulty, 2013) because of the associated difficulty. For example, using subjective experiments, many parameters such as the mechanical properties, the transmittance of nerve signals, and individual feelings are intricately mixed; therefore, it is difficult to identify the effects of individual elements and the extent of their effects.

In this study, we developed a two-dimensional cross-section human finger finite element model based on previous studies. Using the developed model, we estimated the influence of age-related changes in skin stiffness on a mechanical state around Merkel cells. Moreover, this analysis presumed tendencies related to age-related changes in tactile perceptions with changing skin stiffness. Then, we compared the presumed tendencies with results obtained 
experimentally by previous studies, and investigated how age-related changes in skin stiffness are related to changes in tactile perceptions that occur with aging.

\section{Methods}

\subsection{Structure of the finite element model}

As mentioned, the skin consists of three layers (i.e., epidermis, dermis, and subcutaneous tissue). Moreover, fingerprints and dermal papillae might enable tactile receptors to perceive the stimulus effectively (Maeno et al., 1998; Gerling, 2010; Gerling and Thomas, 2005). Therefore, a finger model involving epidermis, dermis, subcutaneous tissue, fingerprints, dermal papillae, nail, and bone was developed. Stratum corneum was not described in this model. The external shape of the fingertip and the thickness of each skin layer used in the model were adopted from Maeno et al. (1998). The size and shape of finger and the thickness of skin layers adopted in the model were obtained from dissected human fingers measured by Maeno et al. (1998). Figure 2 shows the two-dimensional cross-sectional fingertip model used in this study. The model was divided into finite elements (i.e., 8-node quadratic plane strain elements). There were approximately 49,000 elements, and there were approximately 276,000 degrees of freedom. Much finer elements were adopted around the dermal papillae than the model of Maeno et al. (1998) for more detailed analysis of tactile mechanisms.

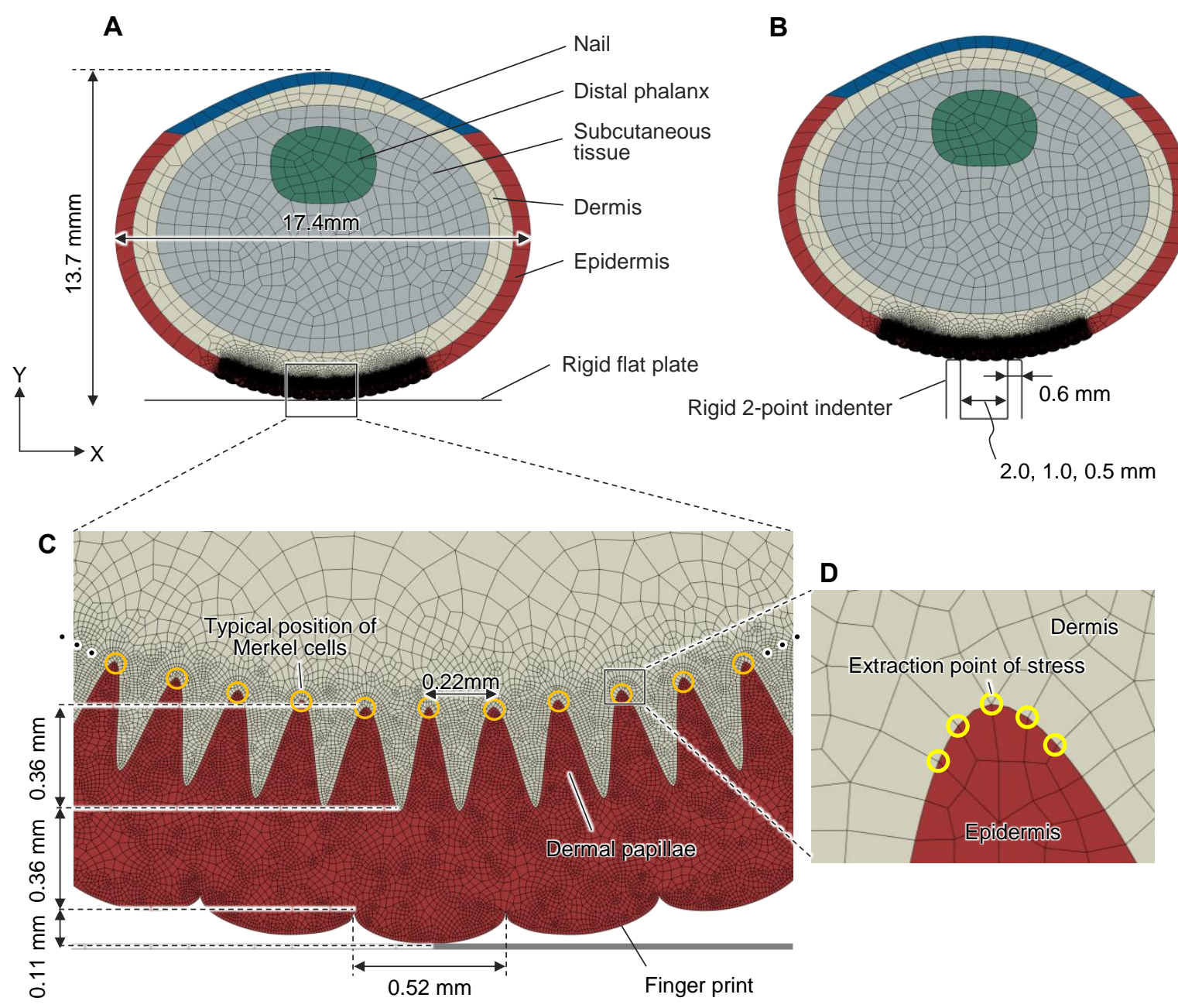

Fig. 2 Fingertip model composed of the epidermis, dermis, subcutaneous tissue, bone (distal phalanx), and nail including papillae. (A) Over all view including the rigid flat plate. (B) Over all view including the rigid 2-point indenter. (C) Details of the dermal papillae and finger print. Circles show the typical position of Merkel cells. (D) Extraction point of stress. 


\subsection{Skin material properties}

Adoption of a linear elastic model or nonlinear elastic model was considered for the skin tissue model. It has been shown that results of linear elastic models are in good agreement with experimental results using calculation conditions similar to those used for this research (Srinivasan and Dandekar, 1996; Dandekar et al., 2003; Gerling, 2010; Shimojo et al., 2014). Therefore, the skin tissue was represented by linear elastic material. Each material property is shown in Table 1 (Maeno et al., 1998; Shao et al., 2010). Hereafter, the model using these material properties is referred to as the standard (STD) model.

The skin moisture content of human skin decreases with aging, and the epidermis becomes hard (Kuwazuru et al., 2008). Subcutaneous tissue is mainly composed of fat and is separated and compartmented by collagen fibers (Tanaka et al., 2015). In addition, it is generally known that fat decreases (Tchkonia et al., 2010; Palmer and Kirkland, 2016) and the collagen fiber bundle narrows with aging. That is, if subcutaneous tissue is regarded as a uniform solid like in the present model, then subcutaneous tissue tends to soften with aging. In this study, our goal was to estimate the influence of age-related changes in skin stiffness on a mechanical state around Merkel cells and tactile perceptions. Therefore, for comparison, we calculated the stress around the Merkel cells using a model we developed called the changing skin stiffness (CSS) model, which comprised epidermis that was 3-times harder than that of the STD model and subcutaneous tissue that was 3-times softer than that of the STD model. Young's moduli and Poisson's ratios of the STD and CSS models were assumed by referring to the literature (Maeno et al., 1998; Kuwazuru et al., 2008; Amaied et al., 2015). The adopted material properties are shown in Table 1. These values assumed for the STD and CSS models were within the range of Young's moduli reported in some experimental studies obtained from the literature (Derler et al., 2012; Kuilenburg et al., 2012; Li et al., 2012), in which the Young's moduli of epidermis are from $0.001 \mathrm{MPa}$ to 1.5 $\mathrm{MPa}$, those of the subcutaneous tissue are from $0.00012 \mathrm{MPa}$ to $0.05 \mathrm{MPa}$.

Table 1 Material properties of the fingertip model.

\begin{tabular}{lccc}
\hline \hline & \multicolumn{2}{c}{ Young's modulus (MPa) } & \multirow{2}{*}{ Poisson's ratio (-) } \\
\cline { 2 - 3 } & Standard (STD) & Changing skin stiffness (CSS) & \\
\hline Epidermis & 0.136 & 0.408 & 0.48 \\
\hline Dermis & 0.08 & 0.08 & 0.48 \\
\hline Subcutaneous tissue & 0.034 & 0.011 & 0.48 \\
Distal phalanx & 17,000 & 17,000 & 0.3 \\
\hline Nail & 170 & 170 & 0.3 \\
\hline \hline
\end{tabular}

\subsection{Simulation and analysis}

In this study, we focused on the basic motion of touching an object and considered age-related tactile perception evoked by this motion. Therefore, as a boundary condition, static displacement in the negative y-axis direction of a distal phalanx was performed, and the finger pad was in contact with the object. The enforced displacement was $-1 \mathrm{~mm}$ (Maeno et al., 1998). A rigid flat plate or a 2-point indenter was applied as an object body. The flat plate is shown in Fig. 2(A), and the 2-point indenter is shown in Fig. 2(B). The frictional coefficient between the skin and the object was 0.5. A commercially available finite element analysis software (Abaqus) was used for the structural analysis.

Slowly adapting type 1 units (Merkel cells) fired nerve impulses while slowly touching a surface. According to previous studies (Srinivasan and Dandekar, 1996; Dandekar et al., 2003), nerve impulses exerted in human tactile receptors were interrelated with strained energy density near the receptors in a computational model. Moreover, there was a correlation between strain energy density and Mises stress when using an incompressive material; therefore, some previous studies used Mises stress as an index to evaluate the response of tactile receptors (Maeno et al., 1998; Gerling and Thomas, 2005; Shao et al., 2010). According to their studies, in this study, we also used Mises stress around Merkel cells as an index to evaluate tactile perceptions. Mises stress is represented in Eq. (1).

$$
\bar{\sigma}=\sqrt{\frac{1}{2}\left\{\left(\sigma_{x x}-\sigma_{y y}\right)^{2}+\left(\sigma_{y y}-\sigma_{z z}\right)^{2}+\left(\sigma_{z z}-\sigma_{x x}\right)^{2}+3\left(\sigma_{x y}^{2}+\sigma_{x z}^{2}+\sigma_{y x}^{2}+\sigma_{y z}^{2}+\sigma_{z x}^{2}+\sigma_{z y}^{2}\right)\right\}}
$$


In Eq. (1), $\sigma_{i j}$ showed that the first index, $i$, indicates stress acting on a plane normal to the $i$-axis. The second index, $j$, denotes the direction in which the stress acts.

Circles in Fig. 2(C) show the typical position of Merkel cells. In addition, circles in Fig. 2(D) show extraction points for stress. After the stresses from each node were averaged, we used that value as the evaluation index.

\section{Results}

\subsection{Contact with a flat plate}

Contour plots of Mises stress distributions when the finger pad of the STD model was in contact with a rigid flat plate are shown in Fig. 3. The stress concentration was generated around the Merkel cells at the base of the dermal papillae. This result was consistent with that of a previous study (Maeno et al., 1998).

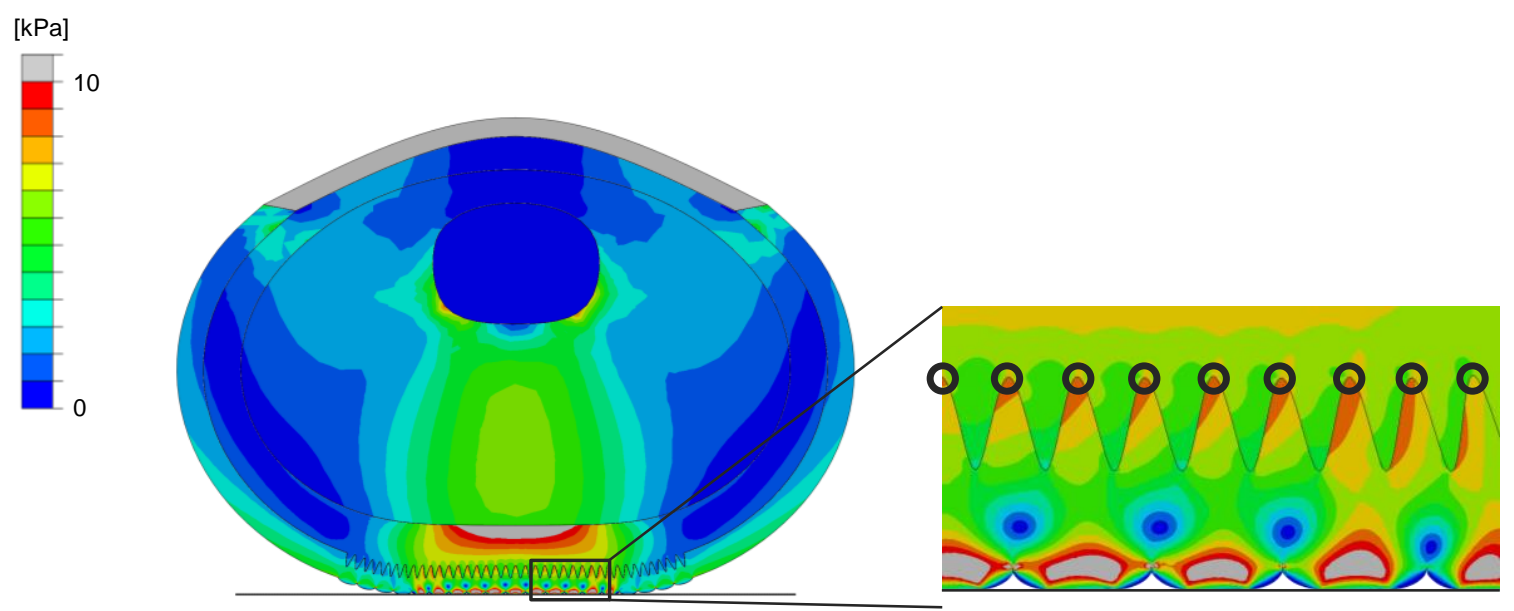

Fig. 3 Mises stress distribution when the finger pad of the STD model is in contact with a rigid flat plate. The stress concentration is generated around the Merkel cells at the base of the dermal papillae (marked with circle), consistent with a previous study (Maeno et al., 1998).

Figure 4(A) shows the deformation of the STD model and Fig. 4(B) shows the Mises stress distribution in areas around the Merkel cells (area marked with a circle in Fig. 2(C)). Figure 4(C) shows the Mises stress distribution found by Maeno et al. (1998). These results were in good agreement, thereby allowing us to confirm the results obtained by the present fingertip model. Therefore, the Mises distribution obtained by the present model was regarded as the response of each Merkel cell (Maeno et al., 1998; Gerling and Thomas, 2005; Shao et al., 2010). For example, in the distribution, a large Mises stress value indicated an active response of the Merkel cell, whereas a small Mises stress value indicated an inactive response of the Merkel cell.

We noticed that the Mises stress distribution was trapezoidal. Moreover, the width of the upper side of the trapezoidal distribution was almost the same as the contact width between the finger pad and the object. These results might indicate why we can perceive exactly how much we touch an object and whether an object's surface is flat when we touch it.

Figure 5 shows the Mises stress distribution of the STD model (solid line) and that of the CSS model assuming aging (dotted line). We realized that the width of the upper side of the trapezoidal Mises stress distribution of the CSS model was narrow compared with that of the STD model. The results indicated that the range of contact with the flat plate became narrow as the stiffness of the skin changed. In fact, the contact range of the STD model was $5 \mathrm{~mm}$, whereas the contact range of the CSS model was $4 \mathrm{~mm}$. The distribution became mountainous instead of trapezoidal. These results suggested that the spatial perception of contact became poor due to the changing skin stiffness that occurs with aging. It is presumed that elderly people whose skin is changed cannot achieve accurate tactile feedback and have incomplete perception when in contact with an object; therefore, they might perform unintended movements. For example, elderly people might excessively push their finger toward an object to compensate for incomplete contact 
recognition. In fact, it was confirmed that elderly people generate greater force than young people during tasks (Olafsdottir et al., 2007; Kapur, 2010).
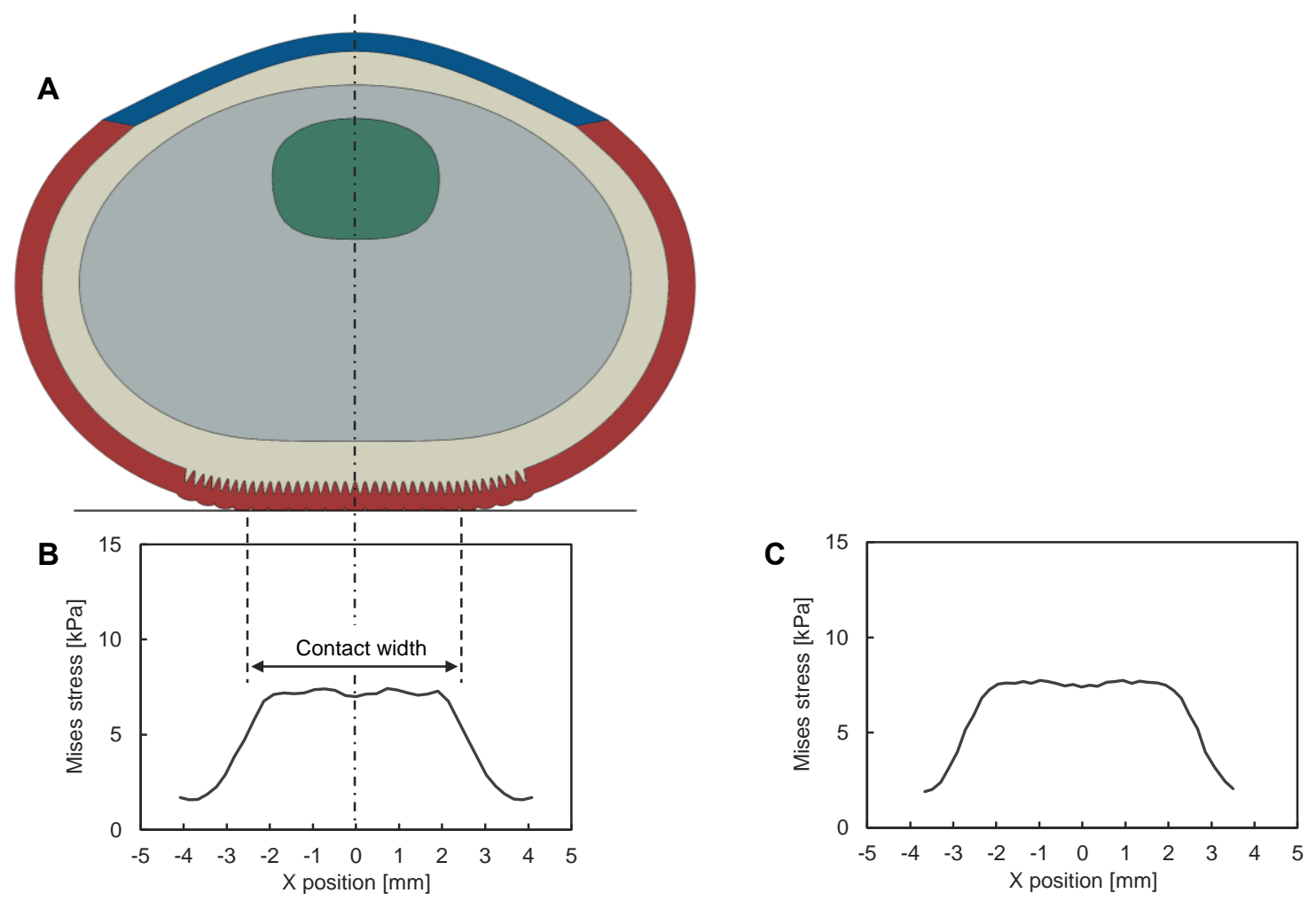

Fig. 4 (A)(B) Deformation of the STD model and the Mises distribution around Merkel cells are shown. The arrow in the figure shows the contact width between the finger pad and flat plate. Mises stress distribution is trapezoidal, and the width of the upper side of the trapezoidal distribution is almost the same as the contact width. (C) The Mises distribution by Maeno et. al. (1998) is shown when the finger pad is in contact with a rigid flat plate. This distribution and the present results are in good agreement.

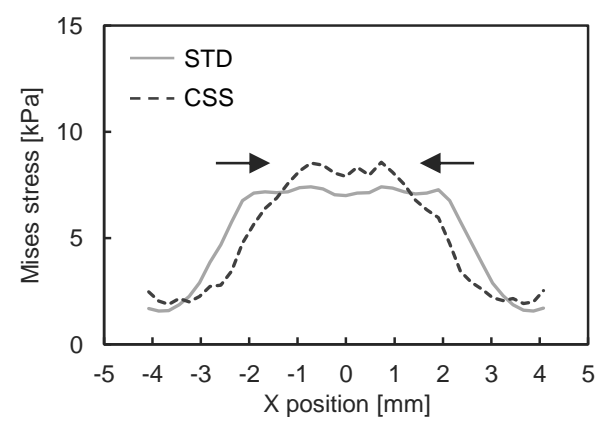

Fig. 5 Mises distribution around Merkel cells is shown. Solid line shows the result of the STD model. Dotted line shows the result of the CSS model. The width of the upper side of the trapezoidal distribution became narrow, and the range of contact with the flat plate became narrow. These results suggest that the spatial perception of contact became poor due to the changing skin stiffness that occurs with aging.

\subsection{Contact with a 2-point indenter}

It has been presented that age-related changes in skin stiffness influence spatial tactile perceptions. This suggestion was confirmed more quantitatively by using a simulation involving a 2-point discrimination test. The 2-point discrimination test has been widely used to measure tactile spatial acuity (Tong and Goldreich, 2013). During the test, a finger pad is stimulated by a 2-point indenter, and the minimum distance that can be distinguished by a gap between the 
2 points is measured. If the discrimination distance between 2 points is less than the measured minimum distance, then we wrongly perceive only 1 point despite being stimulated by the 2 -point indenter. Moreover, the test showed that the minimum 2-point discrimination distance of elderly people is larger than that of young people (Stevens and Choo, 1996; Kalisch et al., 2012; Pleger et al., 2016). The discrimination distance of young people is approximately $2.0 \mathrm{~mm}$, and that of elderly people is approximately $3.5 \mathrm{~mm}$. For example, elderly people could not correctly determine a 2-point gap and wrongly perceive only as 1-point, whereas young people could distinguish the 2-point gap despite being stimulated by the same 2-point indenter. In brief, spatial tactile perceptions that become poor with aging are examined by this 2-point discrimination test. Hence, the influence of age-related changes in skin stiffness on tactile perceptions can be discussed by simulating this 2-point discrimination test.

\subsubsection{The 2-point discrimination test}

First, we confirmed whether the present fingertip model can involve the 2-point discrimination test. Then, we considered how to analyze the obtained results to discuss 2-point discrimination.

Figure 6(A) shows the deformation of the STD model when a finger pad was stimulated by a 2-point indenter. Fig. 6(B)-(D) show the Mises stress distribution around Merkel cells. Figure 6(B) shows the results when the distance between 2 points was $2 \mathrm{~mm}$. Figure 6(C) shows the results when the distance between 2 points was $1 \mathrm{~mm}$. Figure 6(D) shows the results when the distance between 2 points was $0.5 \mathrm{~mm}$.
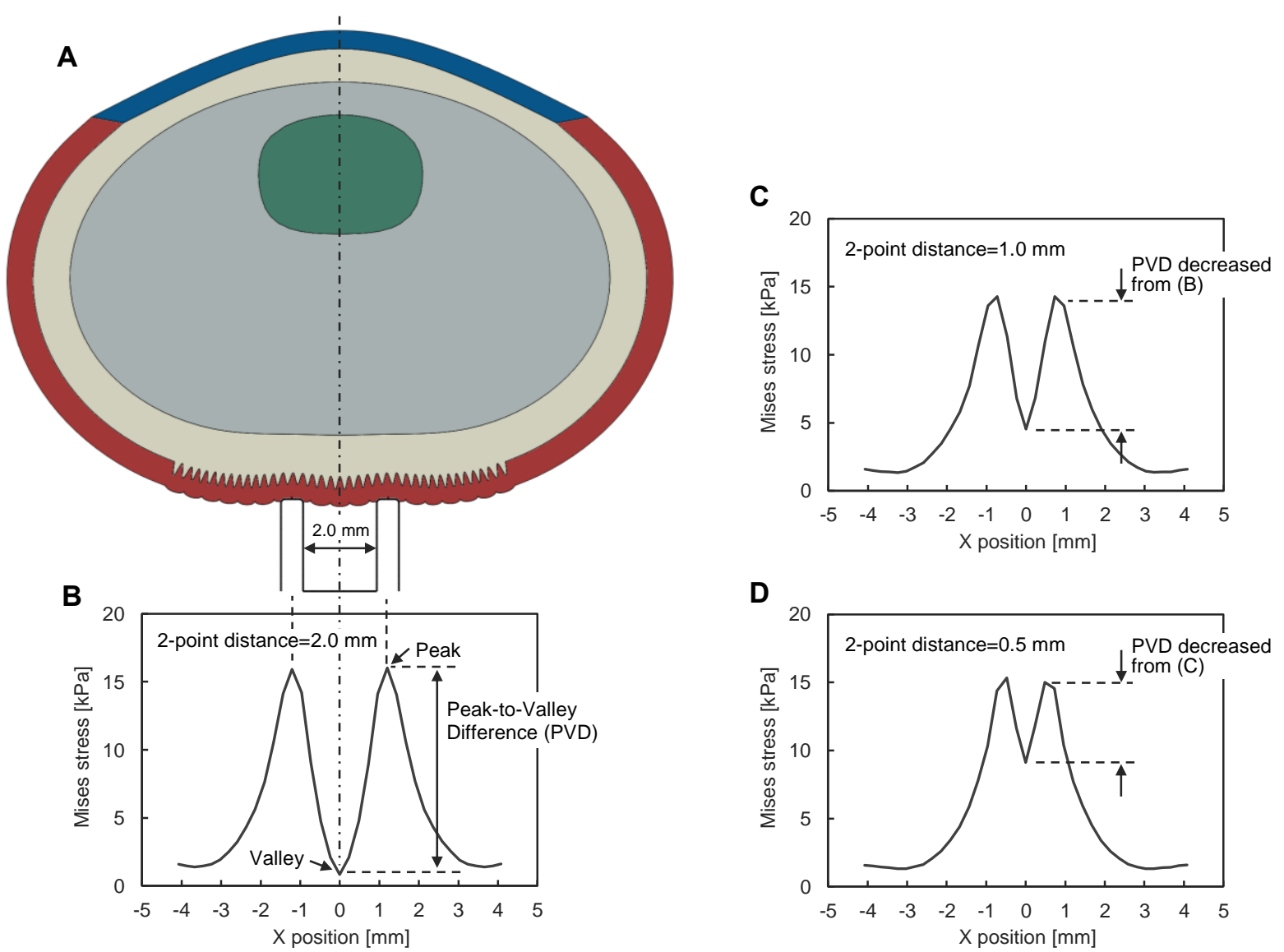

Fig. 6 Deformation of the STD model and the Mises distribution around Merkel cells when the finger pad is in contact with a 2-point indenter are shown. (A) Deformation of the STD model. (B) The distance between the 2 points was $2 \mathrm{~mm}$. (C) The distance between 2 points was $1 \mathrm{~mm}$. (D) The distance between 2 points was $0.5 \mathrm{~mm}$. The valley value of the Mises stress distribution increased, and PVD decreased with the narrowing of the distance between 2 points. When PVD is large, such as in (B), the differences in the responses of Merkel cells are precise; therefore, a gap between 2 points is distinctly perceived. However, when PVD is small, such as in (C) and (D), the differences in the responses of Merkel cells are not clear, and it causes false recognition of the gap. 
Mises stress was maximized beneath the 2 points (Fig. 6(B)). A distribution valley appeared beneath the middle of the 2 points. According to Fig. 6(C) and Fig. 6(D), it was found that the valley value of the Mises stress distribution increased with narrowing of the distance between 2 points. Moreover, it is known that a gap between the 2 points can be distinguished by young people when the distance between 2 points is $2 \mathrm{~mm}$; however, it is difficult to distinguish the gap if the distance becomes narrower than that (Stevens and Choo, 1996). For these results, it is thought that we can distinguish a gap between the 2 points if the difference between the maximum value and the valley value of the Mises stress distribution (peak-to-valley difference [PVD]) (Gerling and Thomas, 2005) is large, such as in Fig. 6(B); however, it is difficult to distinguish the gap, and only a 1-point gap is wrongly perceived if PVD is small, such as in Fig. 6(C) and Fig.6(D). Regarding the Mises distribution as a response of each Merkel cell, the differences in responses are precise when PVD is large; therefore, a gap between 2 points is distinctly perceived. When PVD is small, the differences in the responses of Merkel cells are not clear, and it causes false recognition of the gap. Hence, it is thought that 2-point discrimination can be discussed by estimating PVD.

\subsubsection{Influence of the changes in skin stiffness}

Next, the influence of age-related changes of skin material properties on 2-point discrimination was discussed using PVD.

The Mises stress distribution around Merkel cells when a finger pad was stimulated by a 2-point indenter with CSS assuming aging is shown in Fig. 7 (dotted lines). Figure 7(A) shows the result when the distance between 2 points was $2 \mathrm{~mm}$, and Fig. 7(B) shows the result when the distance between 2 points was $3.5 \mathrm{~mm}$. Solid lines are represented in Fig. 6(B).

A

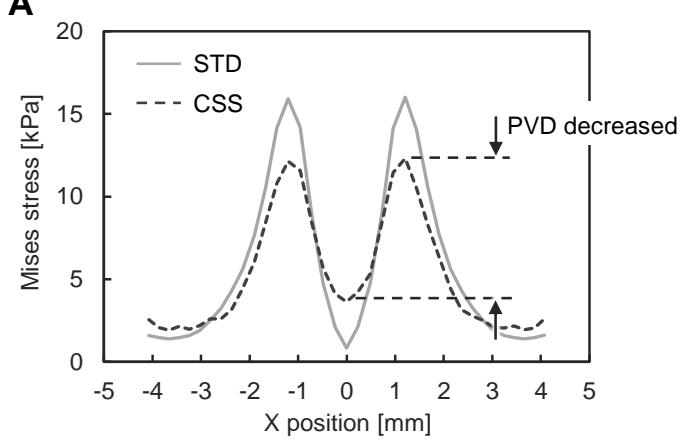

B

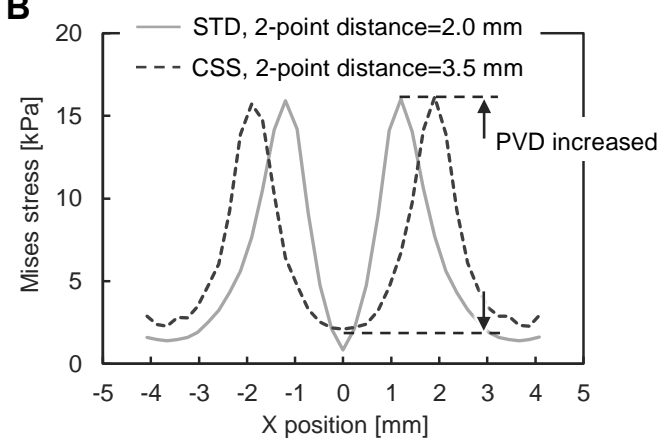

Fig. 7 Mises distribution around the Merkel cells is shown. (A) The distance between the 2 points was 2 mm. The solid line shows the results of the STD model. The dotted line shows the results of the CSS model. PVD of the CSS model decreased from that of the STD model. (B) The solid line shows the results of the STD model (2-point distance was $2 \mathrm{~mm}$ ). The dotted line shows the results of the CSS model (2-point distance was $3.5 \mathrm{~mm}$ ). The CSS model had PVD similar to that of the STD model throughout expansion of the distance between 2 points. These results show the tendency of age-related changes in 2-point discrimination such as previous experimental data (Stevens and Choo, 1996; Kalisch et al., 2012; Pleger et al., 2016).

We realized that PVD of the CSS model decreased by $43 \%$ compared to the STD model (Fig. 7(A)). In the case of CSS, it was presumed that it was difficult to distinguish a gap between 2 points. Moreover, the CSS model had PVD similar to that of the STD model throughout expansion of the distance between 2 points (Fig. 7(B)). These results explain that we can distinguish a gap between the 2 points after expanding the distance between those 2 points when skin stiffness has changed. This tendency agrees with the physiological knowledge of age-related changes in 2-point discrimination. Previous studies revealed that the perceived distance between 2 points by elderly people was larger than that perceived by young people during the 2-point discrimination test (Stevens and Choo, 1996; Kalisch et al., 2012; Pleger et al., 2016). Hence, the present study has demonstrated that age-related changes in skin stiffness influence spatial tactile perceptions.

Incidentally, why did PVD decrease with changing skin stiffness? We analyzed the stress applied around the 
Merkel cells. The dotted line in Fig. 8(A) shows the normal stress in the x-axis direction $\left(\sigma_{x x}\right)$, and the solid line in the figure shows the normal stress in the y-axis direction $\left(\sigma_{y y}\right)$. These results occurred with STD model simulations. Similarly, the normal stresses that occurred with the CSS model are shown in Fig. 8(B). Mises stresses in Fig. 7(A) are also shown in Figs. 8(C) and (D).

Under STD conditions (Fig 8(A)), $\sigma_{x x}$ and $\sigma_{y y}$ were almost the same beneath the middle of the 2 points. In addition, $\sigma_{z z}$ was also the same as $\sigma_{x x}$, and $\sigma_{x y}$ was zero. This means that the value of the Mises stress becomes zero, as seen on the right side of Eq. (1). In other words, Merkel cells (i.e., mechanoreceptor) do not respond in this case because the deformation does not occur due to incompressive conditions. Meanwhile, the difference between $\sigma_{x x}$ and $\sigma_{y y}$ (marked as I in Fig. 8(B)) beneath the middle of the 2 points was large under CSS conditions. This is because the compressive force in the $\mathrm{x}$-axis direction $\left(\sigma_{x x}\right)$ decreased (marked as II in Fig. 8(B)), although the compressive force in the $y$-axis direction $\left(\sigma_{y y}\right)$ was almost the same compared to STD conditions. Besides, peaks of $\sigma_{y y}$ beneath the 2 points decreased (marked as III in Fig. 8(B)) under CSS conditions. These results indicate that PVD decreased with changing skin stiffness because the valley value of the Mises stress increased (marked as IV in Fig. 8(B)), and the peak value of the Mises stress decreased (marked as V in Fig. 8(B)). Additionally, the decreases in $\sigma_{x x}$ (marked as II in Fig. 8(B)) and $\sigma_{y y}$ (marked as III in Fig. 8(B)) were caused not only by the hardening epidermis but also by the softening subcutaneous tissue. Moreover, the factors in the increase of the valley value of the Mises stress (marked as IV) beneath the middle of 2-point indenter were interesting. Normally, we might think that the factor increasing the valley value of Mises stress beneath the middle of the 2 points is the increase in the $\sigma_{y y}$ beneath the middle of the 2 points because the fingertip is compressed in the y-axis direction. However, the factor was the decrease in $\sigma_{x x}$ in the direction of fingertip width (marked as II in Fig. 8(B)). This consideration was not able to be derived by the physiological experiment because the internal mechanical state of the fingertip was not observed, although it can be analyzed by using a numerical analysis.
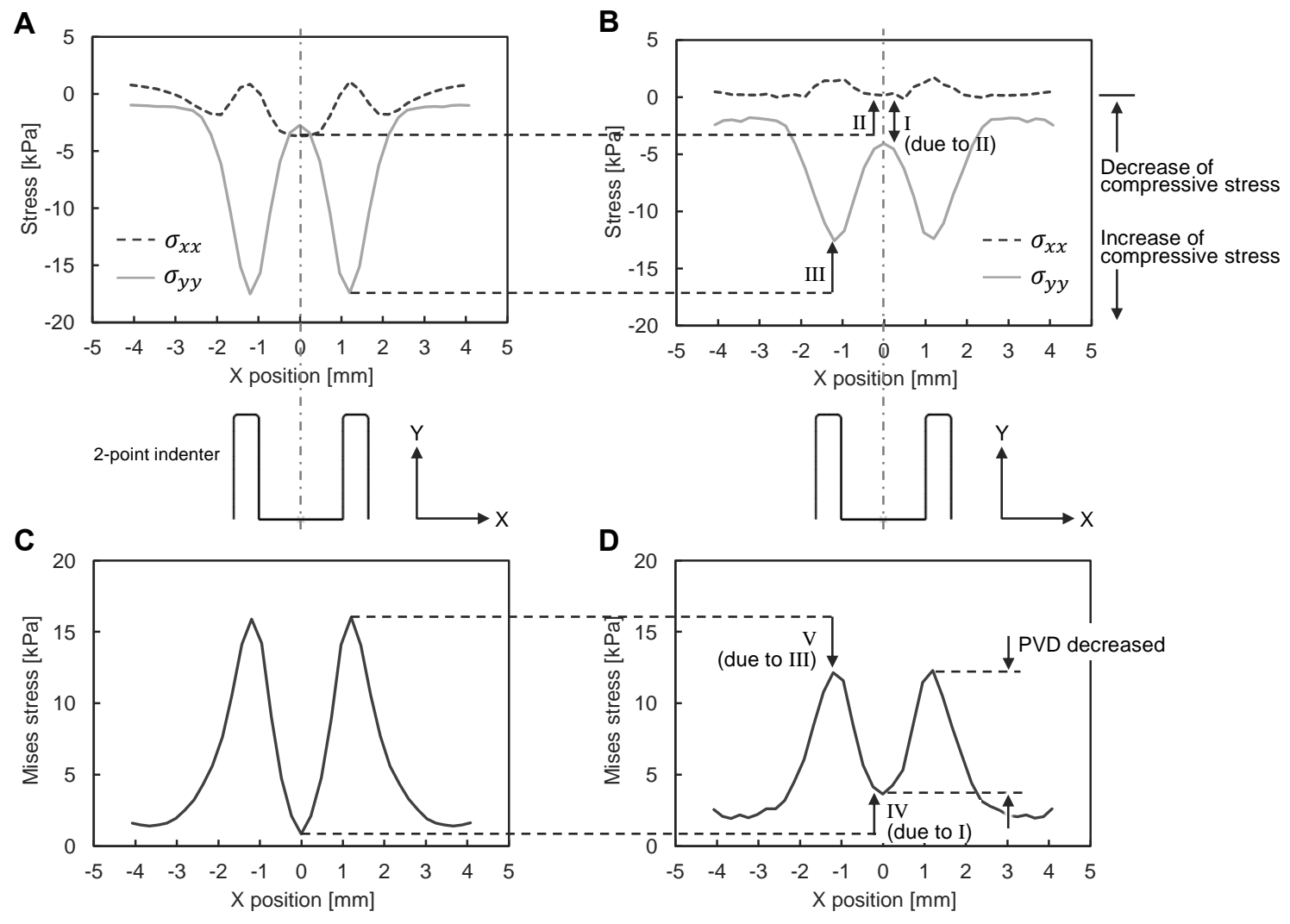

Fig. 8 Analyzing the decrease in PVD with changing skin stiffness around the Merkel cell. (A)(C) Normal stresses in the $\mathrm{x}$-axis and $\mathrm{y}$-axis directions and Mises stress according to simulations using the STD model. (B)(D) Normal stresses in the $\mathrm{x}$-axis and $\mathrm{y}$-axis directions and Mises stress according to simulations using the CSS model. PVD decreased due to the decrease of $\sigma_{x x}$ (marked as II) beneath the middle of the 2-point indenter and the decrease of $\sigma_{y y}$ (marked as III) beneath the 2 points with changing skin stiffness. 


\section{Discussion}

In this study, a two-dimensional cross-section human finger finite element model based on previous studies was developed. Using the developed model, simulations of the fingertip model contacted with a rigid flat plate or a 2-point indenter were performed. The obtained results showed tendencies of age-related changes in skin stiffness leading to dull perceptions, thereby agreeing with physiological knowledge presented in previous studies.

The model used in this study did not describe the stratum corneum. This is because some researchers reported that simulation results using their skin models excluding the stratum corneum had a correlation with experimentally measured nerve discharge frequency (Gerling, 2010; Shimojo et al., 2014). However, the stratum corneum has high stiffness, and its behavior might affect the deformation of skin as well as tactile perceptions. Therefore, we performed some simulations using the STD and CSS models with and without the stratum corneum, in which the thickness of the stratum corneum was set to $0.173 \mathrm{~mm}$ (Egawa et al., 2007) and Young's moduli of the stratum corneum in the STD and CSS models were assumed as 6.0 and 12.0 MPa, respectively (Kuwazuru et al., 2008). Figure 9 shows comparisons of Mises stress distribution around Merkel cells when a finger pad was stimulated by a 2-point indenter under the condition similar to Fig. 6(B), Fig. 6(D) and Fig. 7(A). From Fig. 9(A) and Fig. 9(B) under STD condition, PVD of the model with the stratum corneum in 2.0 and $0.5 \mathrm{~mm}$ gap condition were $14 \%$ and $56 \%$ smaller than that without the stratum corneum, respectively. It seems that the stratum corneum had more effects on PVD under the smaller gap conditions such as $0.5 \mathrm{~mm}$. From Fig. 9(C) and Fig. 9(D) under CSS condition, PVD of the model with the stratum corneum was $17 \%$ smaller than that without the stratum corneum, and the maximum and valley values of the Mises stress of the model with the stratum corneum were $1.04 \mathrm{kPa}$ and $2.38 \mathrm{kPa}$ larger than those of the model without the stratum corneum, respectively. These simulation results suggest that the stratum corneum and age-related changes in the stiffness could affect tactile perceptions. Modeling the stratum corneum will be one of the effective methods in order to improve the accuracy in the estimation of tactile perceptions using PVD.
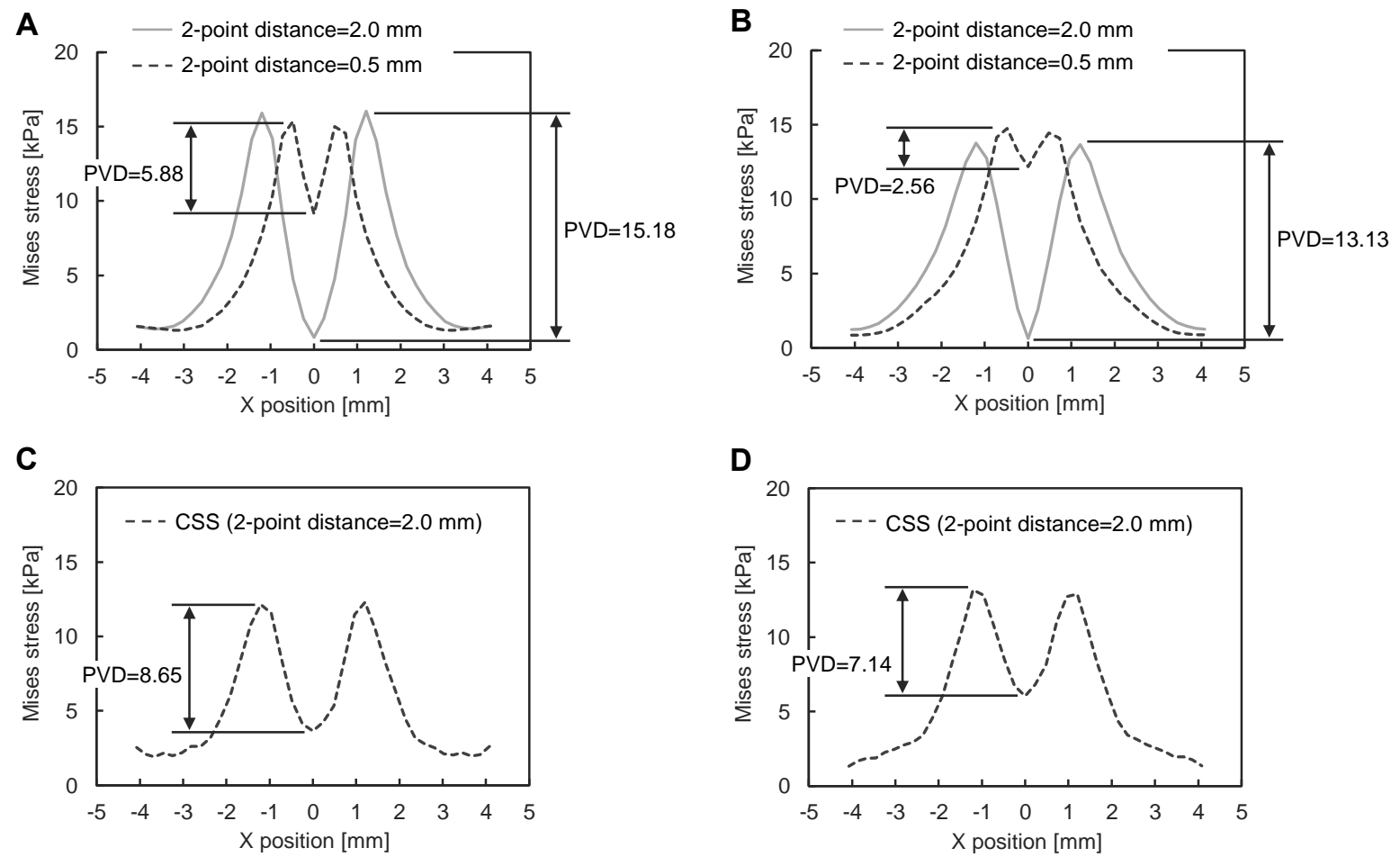

Fig. 9 Comparisons of Mises stress distribution around Merkel cells with and without the stratum corneum. (A)(C) Not considering the stratum corneum. (B)(D) Considering the stratum corneum. (A)(B) Effect on STD condition. (C)(D) Effect on CSS condition.

In the results of 2-point discrimination, the differences of the maximum values of Mises stress in Fig. 6 were 10\%. It may be affected by the position of contact with indenters. Although the differences did not affect qualitative 
evaluations in this study, further studies are needed to investigate how the difference of $10 \%$ affects somatosensory information processing in the brain for quantitative evaluations in tactile perceptions.

Comparing the STD and CSS models, the differences in applied stress around Merkel cells when the same displacement was enforced were compared. Then, it might be thought that the values of stress cannot be compared directly between the two models because the forces applied by the enforced displacement were different between the two models. However, the values of stress were extracted from the elements of the dermis around Merkel cells which did not change the Young's modulus between the STD and CSS models. Therefore, the differences in stress of the dermis between the STD and CSS models are supposed to be caused by mechanical responses of the epidermis and the subcutaneous tissue for the displacements. In addition, in this study, Mises stress and equivalent strain, for example, show qualitatively similar distribution waveforms due to a liner relation between stress and strain of the model using a liner elastic material. From these facts, we believe that we can discuss the simulation results obtained in this study by using Mises stress.

This study had some limitations. Firstly, specific skin material properties that were assumed with referring to previous studies were adopted to represent age-related changes in skin stiffness for static stimulations. In future works, simulations will be performed by treating changes in all skin material properties measured in people of various ages. Moreover, further studies are also needed to investigate the influence of age-related changes in skin viscosity on tactile perception under dynamic stimulation with considering dynamic response of subcutaneous tissues and stratum lucidum. Secondly, changes in skin stiffness were focused in this study. However, tactile perceptions might be influenced by not only skin stiffness but also the other factors. For example, the dehydration of the skin with aging may affect the frictional coefficient, and the shape of fingerprint, that is, the depth of furrows is likely to decrease with aging. Additionally, the density of tactile receptors and somatosensory information processing in the brain also may affect tactile perceptions. These factors should be considered when the influence of overall changes of finger characteristics with aging on tactile perceptions is investigated in the future works.

\section{Conclusion}

In this study, the influence of age-related changes in skin stiffness on tactile perception for static stimulations was estimated by a two-dimensional cross-section human finger finite element model. The fingertip finite element models with different stiffness of epidermis and subcutaneous tissues were used to simulate Mises stress distribution around Merkel cells under boundary conditions of contacts with a rigid flat plate or a 2-point indenter. Simulation results with a rigid flat plate showed that the contact width between the finger pad and the object was $5 \mathrm{~mm}$ in the STD model while that changed from $5 \mathrm{~mm}$ to $4 \mathrm{~mm}$ in the CSS model. Simulation results with a 2-point indenter showed that PVD in the CSS model decreased by $43 \%$ comparing to the STD model when it is hypothesized that PVD of the Mises distribution around the Merkel cells could describe 2-point discrimination. These results indicated that age-related changes in skin stiffness had tendencies to influence tactile perceptions. Further studies are required for qualitative investigations on influence of density of tactile receptors, dynamic stimulation, and somatosensory information processing in the brain, and quantitative investigations with considering the other factors related to aging such as the dehydration of the skin, the shape of fingerprint, and the stiffness of the stratum corneum.

\section{References}

Amaied, E., Vargiolu, R., Bergheau, J. M. and Zahouani, H., Aging effect on tactile perception: Experimental and modelling studies, Wear, 332-333 (2015), pp.715-724.

Bowden, J. L. and McNulty, P. A., Age-related changes in cutaneous sensation in the healthy human hand, Age, Vol.35(4) (2013), pp.1077-1089.

Carmeli, E., Patish, H. and Coleman, R., The aging hand, The Journals of Gerontology. Series A, Biological sciences and medical sciences, Vol.58(2) (2003), pp.146-152.

Daly, C. H. and Odland, G. F., Age-related changes in the mechanical properties of human skin, Journal of Investigative Dermatology, Vol.73(1), (1979), pp.84-87.

Dandekar, K., Raju, B. I. and Srinivasan, M. A., 3-D finite-element models of human and monkey fingertips to investigate the mechanics of tactile sense, Journal of Biomechanical Engineering, Vol.125, (2003), pp.682-691. 
Derler, S. and Gerhardt, L. C., Tribology of Skin: Review and Analysis of Experimental Results for the Friction Coefficient of Human Skin, Tribology Letters, Vol.45, No.1 (2012), pp.1-27.

Egawa, M., Hirao, T. and Takahashi, M., In vivo Estimation of Stratum Corneum Thickness from Water Concentration Profiles Obtained with Raman Spectroscopy, Acta Dermato-Venereologica, Vol. 87, (2007), pp.4-8.

Gerling, G. J., SA-I mechanoreceptor position in fingertip skin may impact sensitivity to edge stimuli, Applied Bionics and Biomechanics, Vol.7 (2010), pp.19-29.

Gerling, G. J. and Thomas, G. W., The effect of fingertip microstructures on tactile edge perception, Proceedings of the Eurohaptics Conference (2005), pp.63-72.

Johnson, K., Neural basis of haptic perception, John Wiley \& Sons, (2002), pp.537-583.

Kalisch, T., Kattenstroth, J. C., Kowalewski, R., Tegenthoff, M. and Dinse, H. R., Cognitive and Tactile Factors Affecting Human Haptic Performance in Later Life, PLOS ONE, (2012).

Kapur, S., Zatsiorsky, V. M. and Latash, M. L., Age-related changes in the control of finger force vectors, Journal of Applied Physiology, Vol.109(6) (2010), 1827-1841.

Kuilenburg, J., Masen, M. A. and Heide, E., Contact modelling of human skin: what value to use for the modulus of elasticity?, Journal of Engineering Tribology, Vol.227, No.4 (2012), pp.349-361.

Kuwazuru, O., Saothong, J. and Yoshikawa, N., Evaluation of aging effects on skin wrinkle by finite element method, Journal of Biomechanical Science and Engineering, Vol.3, No.3 (2008), pp.368-379.

Li, C., Guan, G., Reif, R., Huang, Z. and Wang, R. K., Determining elastic properties of skin by measuring surface waves from an impulse mechanical stimulus using phase-sensitive optical coherence tomography, Journal of The Royal Society Interface, Vol.9, No.4 (2012), pp.831-841.

Maeno, T., Kobayashi, K. and Yamazaki, N., Relationship between the structure of human finger tissue and the location of tactile receptors, JSME International Journal Series C Mechanical Systems, Machine Elements and Manufacturing, Vol.41, No.1 (1998), pp.94-100.

Olafsdottir, H., Zhang, W., Zatsiorsky, V. M. and Latash, M. L., Age-related changes in multifinger synergies in accurate moment of force production tasks, Journal of Applied Physiology, Vol.102(4) (2007), 1490.

Palmer, A. K. and Kirkland, J. L., Aging and adipose tissue: potential interventions for diabetes and regenerative medicine, Experimental Gerontology, Vol.86 (2016), pp.97-105.

Pleger, B., Wilimzig, C., Nicolas, V., Kalisch, T., Ragert, P., Tegenthoff, M. and Dinse, H. R., A complementary role of intracortical inhibition in age-related tactile degradation and its remodelling in humans, Scientific Reports(Nature), 6, Article number 27388, (2016).

Saxon, S. V., Etten, M. J. and Perkins, E. A., Physical change and aging, Springer Publishing Company, (2010), pp.21-23.

Shao, F., Childs, T. H. C., Barnes, C. J. and Henson, B., Finite element simulations of static and sliding contact between a human fingertip and textured surfaces, Tribology International, Vol.43, Issue 12 (2010), pp.2308-2316.

Shimojo, M., Maeno, H., Shinoda, H. and Sano, A., Tactile recognition mechanism and applied technology, S\&T Book (2014), pp.70 (in japanese).

Srinivasan, M. A. and Dandekar, K., An investigation of the mechanics of tactile sense using two-dimensional models of the primate fingertip, Journal of Biomechanical Engineering, Vol.118, (1996), pp.48-55.

Stevens, J. C., Aging and spatial acuity of touch, The Journal of Gerontology, Vol.47, No.1 (1992), pp.35-40.

Stevens, J. C. and Choo, K. K., Spatial acuity of the body surface over the life span, Somatosensory \& Motor Research, Vol.13 (1996), pp.153-166.

Tanaka, Y., Ito, T., Hashimoto, M., Fukasawa, M., Usuda, N. and Sano, A., Collagen fibers induce expansion of receptive field of Pacinian corpuscles, Advanced Robotics (2015), pp.735-741.

Tchkonia, T., Morbeck, D. E., Zglinicki, T., Deursen, J., Lustgarten, J., Scrable, H., Khosla, S., Jensen, M. D. and Kirkland, J. L., Fat tissue, aging, and cellular senescence, Aging Cell, Vol.5 (2010), pp.667-684.

Tong, J., Mao, O. and Goldreich, D., Two-point orientation discrimination versus the traditional two-point test for tactile spatial acuity assessment, Frontiers in Human Neuroscience, Vol.7, (2013), Article 579.

Vallbo, A. B. and Johansson, R. S., Properties of cutaneous mechanoreceptors in the human hand related to touch sensation, Human Neurobiology, Vol.3, (1984) pp.3-14.

Wickremaratchi, M. M. and Llewelyn, J. G., Effects of ageing on touch, Postgraduate Medical Journal, Vol.82(967) (2006), pp.301-304. 